\title{
Medication errors in nursing homes: the role of pharmacological knowledge.
}

\author{
Sam Kosari* \\ Discipline of Pharmacy, Faculty of Health, University of Canberra, Bruce, ACT, Australia
}

Keywords: Medication error, Nursing homes, Nursing staff, Pharmacological knowledge.

Accepted on January 2021

\section{Introduction}

In nursing home settings, medication errors have been an ongoing problem $[1,2]$. Early studies reported a medication administration error rate of $12.2 \%$ of the total doses of medication in a sample of 52 nursing home throughout the U.S [1]. A recent systematic review also revealed that, internationally, medication errors were commonly observed in $16-27 \%$ of nursing home residents [2]. Administration of the incorrect dosage of medications was the most prevalent type of medication error and most likely cause of harm to residents [2].

Nursing staff are involved in medication administration more than other health care professionals and are identified as major contributors to medication errors [3]. A cross-sectional study [4] that reported the views of nurses on medication errors concluded that lack of pharmacological knowledge is the leading cause of medication errors by nursing staff. Lack of familiarity with drugs' generic and brand names, doses and pharmacological properties of drugs can create confusion, especially with those drugs that sound or look alike.

Confusing drug names is one of the most common factors contributing to medication errors as identified by the World Health Organization [5]. Additionally, many drugs' packaging or containers look similar, and they may be located next to each other in hospitals, pharmacies or nursing homes [5]. For example, a recent case report from Australia [6] reported inadvertent administration of a non-ocular pharmaceutical product (mometasone lotion) into the eye of a nursing home resident due to similarities in the shape and size of the packaging and the products being placed next to each other by nursing staff. Further investigation showed that these errors are quite common and not limited to nursing home settings. Interestingly, a review of calls made to an Australian poisons information centre over a 7-year period showed that mometasone lotion was the most common pharmaceutical product accidentally instilled into the eye [7]. This indicates several system failures, such as storage of drugs, labelling of the products, packaging similarities and limited pharmacological knowledge of nursing staff.

To reduce medication administration errors and to ease the burden of medication management for nurses, most nursing homes in Australia use dose administration aid (DAA) devices that are prepared by pharmacies [8]. DAAs are used to organize oral medications according to the day of the week and time of day that they must be taken [9]. These devices are reported to save time and reduce errors in medication administering in nursing homes [8]. Using DAAs facilitates delegating some basic medication administrations tasks to personal care workers, who are not required to complete the extensive medicine training required for nurses [8]. However, DAAs can only be used for solid dosage forms such as tablets and capsules; they are not suitable for many other types of medications, such as liquids, semi-solid preparations, dispersible or effervescent tablets, moisture-sensitive medications and topical products [10]. Thus, it is equally important for both nurses and personal care workers who are involved in medication administration to have relevant pharmacological knowledge of the medications that they administer.

Lack of pharmacological knowledge of nursing and care staff can be addressed by strategies such as collaborative learning activities with pharmacists or nurse educators [11], regular continuing professional development activities [12], on-site training about common medications they administer [11], facilitating access to pharmacology and medication information resources [13] and developing a systematic approach to respond to medication error incidences that have occurred, by providing sufficient training and education to prevent further similar incidences [4,14]. There is also a need for further research to identify evidence-based strategies to address the gap in the pharmacological knowledge of nursing staff, to reduce medication error incidences and improve patient safety.

\section{References}

1. Barker KN, Mikeal RL, Pearson RE, et al. Medication errors in nursing homes and small hospitals. Am J Hosp Pharm. 1982;39(6):987-91.

2. Ferrah N, Lovell JJ, Ibrahim JE. Systematic Review of the Prevalence of Medication Errors Resulting in Hospitalization and Death of Nursing Home Residents. J Am Geriatr Soc. 2017;65(2):433-442.

3. Hughes RG, Blegen MA. Medication administration safety. Patient safety and quality: An evidence-based Handbook for nurses. Rockville (MD) 2008.

4. Cheragi MA, Manoocheri H, Mohammadnejad E, et al. Types and causes of medication errors from nurse's viewpoint. Iran J Nurs Midwifery Res. 2013;18(3):228-31.

5. Centre for Patient Safety Solutions, WH.O.C. Look-alike sound-alike medication names. Patient Safety Solutions. 1:20075

6. Naunton M, Kowsar Nor, Bartholomaeus A, et al. Case report of a medication error: In the eye of the beholder. Medicine (Baltimore). 2016;95(28):e4186.

7. Brown JA. Medicinal mishap: Incorrectly dropped in the eye. Aust Prescr 2013;36:56-7.

8. Gilmartin, JF, Hussainy SY, Marriott JL. Medicines in Australian nursing homes: a cross-sectional observational 
Citation: Kosari S. Medication errors in nursing homes: the role of pharmacological knowledge. J Intensive Crit Care Nurs. 2021; $\square(1): 1-2$.

study of the accuracy and suitability of re-packing medicines into pharmacy-supplied dose administration aids. Res Social Adm Pharm. 2013;9(6):876-83.

9. Gilmartin, JF, Marriott JL, Hussainy SY. Towards improving dose administration aid supply: a quality improvement intervention aimed at reducing dispensing errors. Int J Clin Pharm. 2013;35(6):1152-60.

10. Guidelines and Standards for Pharmacists. Dose Administration Aids Service Pharmaceutical Society of Australia 2007. Available at: http://www.psa.org.au/ supporting-practice/professional-practice-standards/doseadministration-aids-service

11. Ndosi ME, Newell R. Nurses' knowledge of pharmacology behind drugs they commonly administer. J Clin Nurs. 2009; 18(4):570-80.

12. Cleary-Holdforth J, Leufer $\mathrm{T}$. The strategic role of education in the prevention of medication errors in nursing: part 2. Nurse Educ Pract. 2013;13(3):217-20.
13. Nichols P, Copeland TS, Craib IA, et al. Learning from error: identifying contributory causes of medication errors in an Australian hospital. Med J Aust. 2008;188(5):276-9.

14. Tang F I, Sheu SJ, Yu S, et al. Nurses relate the contributing factors involved in medication errors. J Clin Nurs, 2007;16(3):447-57.

\section{*Correspondence to:}

Sam Kosari

Discipline of Pharmacy,

Faculty of Health, University of Canberra,

Bruce, ACT, Australia

E-mail: sam.kosari@canberra.edu.au 